Itinéraires Itinéraires

Littérature, textes, cultures

2012-3 | 2013

Lire les villes marocaines

\title{
Représentations de Tanger dans La Vida perra de Juanita Narboni d'Ángel Vázquez
}

\author{
Nathalie Sagnes Alem
}

\section{OpenEdition}

Journals

Édition électronique

URL : http://journals.openedition.org/itineraires/957

DOI : 10.4000/itineraires.957

ISSN : 2427-920X

Éditeur

Pléiade

Édition imprimée

Date de publication : 1 juillet 2013

Pagination : 69-79

ISBN : 978-2-343-01183-7

ISSN : 2100-1340

Référence électronique

Nathalie Sagnes Alem, «Représentations de Tanger dans La Vida perra de Juanita Narboni d'Ángel Vázquez », Itinéraires [En ligne], 2012-3 | 2013, mis en ligne le 01 décembre 2012, consulté le 02 mai 2019. URL : http://journals.openedition.org/itineraires/957 ; DOI : 10.4000/itineraires.957

\section{(ब) $\Theta \Theta \Theta$}

Itinéraires est mis à disposition selon les termes de la licence Creative Commons Attribution - Pas d'Utilisation Commerciale - Pas de Modification 4.0 International. 


\title{
Représentations de Tanger dans La Vida perra de Juanita Narboni d’Ángel Vázquez
}

\begin{abstract}
Through the writing of La Vida perra de Juanita Narboni, Ángel Vázquez has linked his name with the city of Tangier. His fictional world cannot be dissociated from this unique urban space and the myths it has engendered. Tangier may thus be portrayed as a whole world in its own right or as a micropole; as a beloved mother figure or one that is hated; as the embodiment of perfect syncretism or the example of a society that is broken in more than one way. It reflects all the fantasies and frustrations of post-colonial society. By focusing his novel on all those contradictions and tensions, after twelve years of silence and exile, Vázquez is thus able to reconnect with the town's memory.
\end{abstract}

Keywords : Tangier, myth-making/unmaking, monologue, memory, identity, postcolonialism

Mots clés: Tanger, mystification/démystification, monologue, mémoire, identité, postcolonialisme

La ville se présente comme un espace de signes à déchiffrer dans le texte lui-même, ce qui nous conduit à interroger le rapport de spécularité que la fiction entretient avec la réalité. Il existe une analogie entre la fondation d'une ville et la genèse d'une œuvre de fiction, si l'on accepte la définition que donne Roland Barthes de cette dernière : la « construction d'un monde autarcique fabriquant lui-même ses dimensions et ses limites, et y disposant son temps, son espace, sa population, sa collection d'objets et ses mythes ${ }^{1} \gg$. La ville en tant qu'espace urbain et la ville poétique ou littéraire ne peuvent

1. Jean-Marie Grassin choisit, pour étayer son propos, de reprendre cette citation de Roland Barthes, extraite du Degré zéro de l'écriture (Paris, Seuil, 1953, p. 25). Il ajoute : «Les espaces humains sont des réalités subjectives définies par la langue qui les dispose et les oppose. La ville n'est pas un objet fixe, mais un espace en continuelle re-définition dans sa conception et dans sa frontière » (dans Juliette Vion-Dury (dir.), L'Écrivain auteur de sa ville, Limoges, Presses universitaires de Limoges, 2001, p. 9). 
s'appréhender séparément, et ceci est d'autant plus vrai dans le cas de Tanger dont les nombreuses représentations artistiques et fictionnelles ont nourri depuis près d'un siècle un imaginaire collectif presque universel ${ }^{2}$.

\section{La ville des origines}

Ángel Vázquez est né en 1929 dans une ville tout juste dotée du fameux statut international ${ }^{3}$ et il ne la quittera qu'en 1965 alors que la plus grande partie de la colonie espagnole et de la communauté européenne qui y résidait s'est déjà exilée. Installé depuis toujours au cœur de la médina, comme nombre de ses compatriotes espagnols d'origine modeste, il côtoie la communauté séfarade qui y vit et fréquente des artistes ou intellectuels de toute nationalité de passage à Tanger ou ayant choisi d'y demeurer pour toujours ${ }^{4}$. En 1962, le prix Planeta lui est attribué pour son premier roman Se encienda y se apaga una luz mais son deuxième roman, Fiesta para una mujer sola ${ }^{6}$, publié en 1964, passe presque totalement inaperçu. Dans ces deux œuvres, où les personnages principaux sont déjà des femmes (Cristina une jeune fille qui sort de l'adolescence pour le premier et Paula une femme d'âge mûr pour le second), la ville de Tanger sert de cadre à l'action mais ses représentations n'échappent pas au cliché. J'entends ici le cliché comme un avatar du mythe qui en questionne cependant la légitimité. L'écrivain semble d'abord avoir du mal à se défaire de stéréotypes (la ville cosmopolite, les rencontres faciles, la permissivité, les affaires douteuses) comme s'il devait combler une attente chez un lecteur en mal d'exotisme facile $^{7}$. La dimension pluriculturelle de la ville ne se donne à voir qu'à travers des personnages secondaires de différentes nationalités. Dans ces deux romans, le rapport à la langue est déjà posé comme un aspect

2. On peut citer les peintres Delacroix et Matisse ou les écrivains tels Paul Bowles, Juan Goytisolo ou Tahar Ben Jelloun pour lesquels la ville a été aussi bien un lieu de vie qu'un objet artistique ou poétique.

3. Le statut international, par lequel les grandes puissances se partagent l'administration de la ville, a été adopté officiellement en 1923 et a duré jusqu'à l'indépendance du Maroc en 1956. 4. Paul Bowles raconte qu'il avait été intrigué par ce petit bonhomme introverti et plein d'humour mais c'est avec son épouse, Jane, que Vázquez nouera une relation amicale privilégiée.

5. Ángel Vázquez, Se enciende y se apaga una luz, Barcelone, Planeta, 1962 (On éteint et on allume une lampe, œuvre non traduite en français).

6. Fiesta para una mujer sola, Barcelone, Planeta, 1964 (Fête pour une femme seule, œuvre non traduite en français).

7. Le jeune amant espagnol de Paula est fasciné par la simple évocation de la ville : «De pronto, sin saber por qué se acordó de las cajas de dátiles y de las telas que a tía Florencia le habían mandado de Tánger, cuando él era pequeño [...] y hasta de una edición de Las mil y una noches » (Fiesta para una mujer sola, op. cit., p. 18). Traduction de l'auteur: «Soudain, sans savoir pourquoi, il se souvint des boîtes de dattes et des tissus qu'on avait envoyés de Tanger à sa tante Florencia, lorsqu'il était petit [...] et même d'une édition des Mille et une nuits. » 
déterminant de la représentation de l'espace tangérois même si la focalisation du discours conduit à ce que la langue des autres communautés ne soit traitée que sous la forme de bribes de dialogues rapportés. La syntaxe originale ne subit pas d'altération et cette restitution du plurilinguisme de la ville rend compte d'une juxtaposition de langues, plutôt que d'une fusion des cultures. La personnalité de chacun s'enrichit sans doute de ces contacts multiples mais, à l'image de la langue première, elle ne fonde pas une nouvelle identité syncrétique. Ces emprunts linguistiques concernent presque toujours le français ou l'anglais, plus rarement l'arabe, mais la hakitia, dont Vázquez fera par la suite la voix symbolique de Tanger ${ }^{8}$, est totalement absente des premiers récits. Cependant, et c'est une évidence pour le lecteur, l'auteur a toujours cherché à écrire sa ville autant par la voix des personnages que par leurs actions.

Dans ces deux romans, l'auteur a donc choisi d'utiliser une focalisation externe qui accentue l'impression, par ailleurs relayée par la diégèse, d'un certain détachement traduisant la profonde crise identitaire du sujet postcolonial. La ville, saisie au lendemain de l'indépendance du Maroc, renvoie aux habitants d'origine européenne une image brouillée de ce qu'elle fut et de ce qu'elle est en train de devenir, reflet de leurs propres incertitudes sur leur place dans ce nouvel espace devenu marocain. On pourrait dire qu'Ángel Vázquez se cherche aussi en tant que romancier et qu'il n'a pas encore trouvé la façon de raconter sa ville et de se raconter lui-même. La relative stérilité des représentations de la ville des premières œuvres révèle un malaise que l'écriture ne lui permet pas encore de transcender. À cet égard, les deux premiers romans sont des ébauches ou plutôt des jalons dans l'accomplissement de l'écrivain. La ville de Tanger est certainement « plus qu'un lieu ${ }^{9}$ » dans les premiers textes, mais elle ne révèle pas toute sa complexité comme ce sera le cas dans La Vida perra de Juanita Narboni. À la mort de sa mère, l'écrivain décide de s'exiler ${ }^{10}$ et il écrit dans une

8. «Varias fueron las lenguas que allí tuvieron uso natural pero, fuera aparte el árabe, a todos dominó un castellano popular - del pueblo - alimentado por la Baja Andalucía y, muy particularmente, por esos hebreos sefarditas, tan inefables como poco conocidos de los españoles, amantes conservadores durante siglos de un castellano arcaico ", prologue de La Vida perra de Juanita Narboni [1976], Barcelone, Seix Barral, 1990, p. 9. Toutes les traductions de cette œuvre proviennent de l'édition française (La Chienne de vie de Juanita Narboni, trad. Selim Cherief, Lyon, Rouge Inside, 2009) dont nous nous contenterons d'indiquer la page : « De nombreuses langues y furent couramment utilisées mais, mis à part l'arabe, la plus répandue était un espagnol populaire - au sens littéral - issu de l'Andalousie méridionale et plus particulièrement du parler des Juifs séfarades - ineffables autant que mal connus des Espagnols - qui ont perpétué avec amour, des siècles durant, l'usage d'un castillan archaïsant $[\ldots] »($ p. 19-20).

9. Jean Tena, « La ville est plus qu'un lieu », Imprévue, nº 2, 1982, p. 145-166.

10. Il est difficile de ne pas opérer une lecture presque psychanalytique de la concomitance qui existe entre les deux événements et d'envisager alors symboliquement la ville comme un espace matriciel. 
lettre datée du 12 juillet 1965 à Emilio Sanz de Soto, son ami de toujours : «Me fuí al Consulado y, el 17 de mayo, zarpé para nunca jamás volver de los brazos de esa puta llamada Tánger ${ }^{11}$. » La violence verbale dont il fait alors preuve à l'encontre de sa ville révèle sans doute son désespoir de la quitter et son angoisse face à l'exil qui l'attend, mais elle traduit aussi une complaisance presque jouissive à se servir une dernière fois de l'image consacrée. En effet, Tanger a toujours fasciné les créateurs et les artistes, et la métaphore, presque une métonymie lorsqu'on se souvient des bordels et des tripots qui ont fait aussi la gloire de la cité ${ }^{12}$, de la villeputain est récurrente ${ }^{13}$. Dans la littérature espagnole, depuis le Romancero ${ }^{14}$ de l'époque de la Reconquête, la ville est toujours personnifiée : femme conquise, séduite ou violée. Elle permet de dire l'histoire et le mythe qui sont avec la langue et le territoire le ciment d'une communauté. Le roman réaliste ou naturaliste entretient, quelques siècles plus tard, une relation tout aussi exclusive avec l'espace urbain omniprésent, dans une double tension à la fois référentielle et métonymique. Les descriptions occupent une place prépondérante dans le dispositif diégétique, et même si la neutralité du romancier n'est qu'un mirage de l'écriture, il s'agit quand même toujours de faire de la représentation un enjeu essentiel du roman. À cet égard, l'incipit du plus célèbre roman espagnol du XIX siècle, La Regenta de Clarín, est exemplaire : il s'ouvre sur l'image de Vetusta, la bien nommée, une ville saisie au moment de la sieste et de la digestion, rêvant de sa gloire passée ${ }^{15}$. Si le Tanger de Juanita peut présenter certaines similitudes avec la ville de Clarín, le système descriptif est en revanche inexistant dans le dernier roman de Vázquez. En effet, les canons de l'esthétique réaliste sont dynamités et déconstruits par le monologue du personnage-narrateur et par la structure fragmentaire de l'œuvre.

\section{La ville : lieu de mémoire}

L'immersion du lecteur dans la conscience du personnage l'oblige à voir la ville à travers son regard, un regard presque myope qui se focalise

11. Traduction de l'auteur : «Je me suis rendu au Consulat et, le 17 mai, j'ai levé l'ancre pour quitter les bras de cette pute que l'on appelle Tanger et n'y jamais revenir. »

12. «Ce n'était pas une cité dortoir Tanger mais une cité bordel» (Alphonse Boudard, Manouche se met à table, Paris, Flammarion, 1975, p. 175).

13. Thierry de Beaucé écrit: "On ne parle plus que de la ville maudite, de Tanger la perdue, comme d'une putain embarquée vers des orients crapuleux et salaces » (La Chute de Tanger, Paris, Gallimard, 1984, p. 275).

14. Le Romancero est un recueil de poèmes épiques espagnols du Moyen Âge.

15. «La heroica ciudad dormía la siesta [...]. Vetusta, la muy noble y leal ciudad, corte en lejano siglo, hacía la digestión del cocido y de la olla podrida » (Clarín, La Regenta [1884], Madrid, Castalia, 1987, p. 83). Traduction de l'auteur : «L'héroïque cité faisait la sieste [...]. Vétusta, la très noble et très loyale cité, ville de cour en un siècle lointain digérait son pot-au-feu et son bouilli. » 
exclusivement sur les détails du quotidien. L'espace qui l'entoure ne fait qu'un avec la narratrice et ce n'est le plus souvent que lorsqu'elle note un changement dans ses habitudes qu'elle est amenée à l'évoquer directement, si bien que la ville n'est que rarement saisie dans une perspective synchronique, mais presque toujours dans une perspective diachronique. Le présent de l'énonciation est celui du quotidien, mais il devient aussi celui de la remémoration; cette conception de la temporalité détermine les représentations de l'espace ${ }^{16}$ tout autant que la focalisation du discours. Les lieux de Tanger sont ainsi le plus souvent des « lieux de mémoire » parce que le personnage-narrateur les voit à travers le prisme du souvenir. Par exemple, on ne trouve aucune allusion aux coutumes vestimentaires de la population musulmane alors que la ville était encore dotée de son statut international mais, après l'indépendance, Juanita déplore le changement de décor :

Se acabaron los velos y los jaiques, y el burnús y la yilaba, todo lo que para nosotros tenía el encanto de lo oriental. Miraeste que llevo delante : los pelos largos no te van, mi vida, cuando se tienen los pelitos como tú, rizados, el progreso resulta un problema ${ }^{17}$.

En fait, ce n'est pas tant l'identité de l'Autre qui est questionnée par la narratrice que la sienne propre construite dans la différence. L'ambiguïté du propos révèle que les Marocains ont pris la place de la communauté européenne jadis dominante, en adoptant symboliquement leur façon de se vêtir. De la même façon, les fêtes musulmanes ne sont jamais décrites " en situation », mais affleurent dans le discours comme souvenir d'un temps révolu :

¡Con lo bonitas que eran vuestras costumbres! Daba gusto ver aquellos desfiles de carrozas cuando llegaba el Mulud, con Carro Burro vestido igualito que Madame Du Barry, haciendo una mala imitación de nuestras costumbres. La imitación que hacéis ahora de nosotros es distinta [...]. En la de antes pretendíais agradarnos. En la de ahora [...] lo que pretendéis es asustarnos $^{18}$.

16. Cette relation de l'espace et du temps renvoie au chronotope qui est « une catégorie basée sur la solidarité du temps et de l'espace dans le monde réel comme dans la fiction romanesque. La notion de chronotope fond les indices spatiaux et temporels en un tout intelligible et concret» (Joëlle Gardes-Tamine et Marie-Claude Hubert, Dictionnaire de critique littéraire, Paris, Armand Colin, 1993, p. 35-36).

17. La Vida perra, op. cit., p. 225-226. «Finis les voiles, les haïks, le burnous, la djellaba, tout ce qui avait pour nous le charme de l'Orient. Regarde-moi celui-là : ça ne te va pas les cheveux longs, mon chou. Quand on a les cheveux tout frisés, c'est difficile de suivre les changements de mode...» (p. 291-292).

18. La Vida perra, op. cit., p. 226. «Quand je pense comme vos fêtes étaient jolies! C'était si beau ces défilés de chars pour le Mouloud. Et Cara Burro déguisé... On aurait vraiment dit Madame du Barry. C'était une espèce de caricature de nos coutumes. Maintenant aussi c'est une imitation mais c'est différent, ce n'est plus pareil [...]. Autrefois, vous vouliez nous faire plaisir. Maintenant [...] vous essayez surtout de nous faire peur» (p. 292). 
Le passé est perçu comme nostalgiquement heureux parce qu'il s'oppose aux angoisses que l'indépendance a suscitées dans une partie de la communauté européenne restée dans la ville après 1956. En fait, toute la structure du roman obéit à ce schéma d'une biporalité et de réversibilité des représentations de l'espace urbain. Le roman s'ouvrait sur un meeting aérien auquel assistait la narratrice enfant. Or, cet endroit est devenu le cimetière catholique de la ville ${ }^{19}$. Le prestigieux théâtre Cervantes a également fermé et tombe en ruines tandis que le cinéma Kursaal, que Juanita fréquentait dans sa jeunesse, a été transformé en urinoir municipal, comme s'il s'était agi de faire disparaître les symboles encore visibles de la vie internationale passée. La transfiguration de l'espace tangérois ne fait que rendre visible des bouleversements historiques et sociaux plus profonds que la protagoniste intériorise sans toujours en comprendre les enjeux ${ }^{20}$. Le bal masqué du début du roman donne lieu à une image inversée à la fin de l'œuvre. Tous les signes de la fête sont redistribués et détournés dans le texte : il ne s'agit plus d'évoquer les folles heures de la cité mais la fin d'un monde de pacotille où tout était faux et éphémère comme un jour de carnaval. Le cauchemar s'est substitué à la mémoire dont il questionne désormais la légitimité. Les deux visions, celle d'un passé idéalisé et d'une histoire démythifiée, s'affrontent dans le discours d'un sujet qui a perdu tous ses repères. D'une certaine façon, lorsque la ville devient marocaine, elle cesse d'exister pour la communauté européenne qui préfère la quitter ou la faire symboliquement disparaître dans un gigantesque autodafé ${ }^{21}$.

Les différentes séquences qui composent le roman se déroulent chronologiquement, ce qui implique que la ville est saisie dans un temps historique. Le maillage du roman par l'histoire est confirmé par un certain nombre de références dans la diégèse ${ }^{22}$, mais le monologue s'ancre dans le présent de

19. «Allí iremos todos a parar a Bubana [...]. En Bubana, antes de que instalaran el nuevo cementerio católico [...] vi yo aterrizar por primera vez los aeroplanos » (La Vida perra, op. cit., p. 40). «C'est là que nous finirons tous. À Boubana. C'est là que j'ai vu les trois premiers aéroplanes qui ont atterri ici. C'était avant qu'on crée le nouveau cimetière chrétien » (p. 54).

20. En fait, on pourrait retourner ce que dit Pierre Bourdieu des métamorphoses de l'espace urbain algérien pendant la colonisation et l'appliquer à Tanger après l'indépendance du Maroc : «C'est ainsi que peu à peu l'Européen [le Marocain pour nous] crée un environnement qui lui renvoie son image et qui est la négation de l'univers ancien, un univers où il ne se sent plus étranger, où par un renversement naturel, l'Algérien [1'Européen donc] finit par apparaître comme étranger » (Pierre Bourdieu, Sociologie de l'Algérie [1958], Paris, PUF, 1963, p. 115).

21. «Allí, al fondo, parece como si estuviera ardiendo algo. Ardiendo la ciudad» (La Vida perra, op. cit., p. 246). « Au loin, on aurait dit qu'il y avait un incendie, que la ville était en flammes » (p. 318).

22. Par exemple, lors d'une de ses visites au cimetière, la narratrice informe sa mère de l'entrée des troupes espagnoles dans la ville internationale au lendemain de la défaite des Alliés : «El pasado día doce, a las once de la mañana, entraron las tropas españolas y 
l'énonciation. Cette distorsion temporelle suivant une double temporalité obéit aux codes du discours à la première personne, rend cependant compte de la difficulté du sujet à accepter l'évolution historique de la ville qui, en perdant son statut de ville internationale, devient une ville marocaine. Le monologue apparaît ainsi, symboliquement, comme le mirage d'un présent éternel. L'absence d'évolution du personnage-narrateur (Juanita combat sa solitude et le vide de son existence par des rituels immuables et reste une femme vierge et symboliquement immaculée jusqu'à sa mort) crée un effet de sens redondant.

Le roman s'organise autour d'un axe spéculaire, qui correspond globalement à l'indépendance du Maroc. Pourtant l'événement n'est pas mentionné directement dans la diégèse. Il n'est suggéré que par les craintes qu'il suscite dans la communauté des étrangers qui résident dans la ville ${ }^{23}$ et par ses conséquences. Il est donc éludé du discours (exactement comme la mort de la mère qui ne se donne à voir qu'à partir de la scène de l'enterrement) et devient un non-événement, un «trou noir » dans l'histoire. Cette stratégie de l'ellipse, expression du refoulement chez le personnage, permet de signifier que, dans le monologue intérieur, l'histoire s'appréhende rarement dans l'instant présent et que les silences constituent des signes à interpréter. Lorsque les membres de la communauté européenne se sont pour la plupart dispersés, Juanita continue à refuser à l'Autre, c'est-à-dire au Marocain, la légitimité de sa présence dans la ville, en refusant de le voir et de le nommer ${ }^{24}$. C'est lorsqu'à son tour elle se sent ignorée que la narratrice parvient à mettre en mots les phénomènes d'exclusion générés par un système colonial jamais reconnu comme tel mais qui produisant, à sa disparition, des comportements identiques mais inversés : « [...] pasan por tu lado como si no existieses... Claro, hemos pasado nosotros tantas veces por el lado de ellos como si no existieran ${ }^{25}$. » On remarquera la possibilité

ocuparon la ciudad» (La Vida perra, op. cit., p. 91). «Le 12, à onze heures, les troupes espagnoles sont entrées et ont pris le contrôle de la ville» (p. 119). En fait, c'est le 14 juin 1940, jour où les Allemands entrent dans Paris, que les Espagnols entrent dans Tanger par la route de Tétouan (Dominique Pons, Les Riches Heures de Tanger, Paris, La Table Ronde, 1990, p. 43). Deux jours plus tard, la ville est rattachée au Protectorat espagnol sur le Nord du Maroc. L'imprécision des dates n'affecte pas la dimension référentielle de l'œuvre et obéit même à une certaine logique diégétique; pour la narratrice, l'événement mérite d'être signalé mais il l'affecte bien moins que la fuite de sa sœur avec son amant.

23. « Nos esperan tiempos terribles [...]. Tanger, kaputt» (La Vida perra, op. cit., p. 158). « C'est un drôle d'avenir qui nous attend [...]. Tanger, kaputt» (p. 204).

24. « ¡Mira cómo están las calles, desiertas! » (La Vida perra, op. cit., p. 179). « Regardemoi ça : pas un chat dans les rues » (p. 232). D'ailleurs tous les personnages masculins issus de cette communauté sont désignés par le prénom de Mohamed, véritable nom générique qui identifie l'origine et la religion mais jamais l'individu.

25. La Vida perra, op. cit., p. 226. "On te passe à côté comme si tu n'existais pas... Évidemment, pendant tout ce temps, c'est nous qui avons fait comme s'ils n'existaient pas » (p. 292). 
qu'offre la langue espagnole d'éluder le sujet du verbe, d'où un effet de mise en abyme du sens. À la fin du roman, lorsque le personnage se réveille au milieu de la nuit, il n'est plus capable de faire la différence entre le rêve et la réalité et ne sait plus où il se trouve. Les repères spatio-temporels se voient métaphoriquement brouillés en même temps que la syntaxe du discours s'altère et s'essouffle. La ville de Tanger, jadis centre du monde, n'est même plus un souvenir, à peine un résidu mémoriel en train de disparaître. Le roman se termine lorsque Juanita fait le constat que même sa mémoire lui fait défaut ( ¡ ¿Esta memoria maldita! La repisa, la repisa... ¡Como tengo la cabeza $\left.{ }^{26 !} »\right)$ et réalise qu'elle a oublié les traits du visage maternel. On sait qu'Ángel Vázquez a quitté Tanger à la mort de sa mère et pour lui, les deux traumatismes sont liés, aussi bien dans sa vie que dans les représentations (ou non-représentations) auxquelles ils donnent lieu. Le système descriptif est progressivement saturé de métaphores mortifères : la narratrice, qui a réduit au fil des ans le périmètre de ses déambulations à travers la ville et ne sort finalement presque plus de chez elle, la compare à un gigantesque cimetière et se voit désormais comme une emmurée vivante dans sa maison-tombeau.

\section{Ville et identité}

La ville de Tanger représente un monde et une société issus de la colonisation, même si le mot est historiquement impropre en raison du statut de « zone internationale » voué à disparaître. Elle cherche dans les relectures et réécritures de son passé un sens à un présent rempli d'incertitudes; en fait, ce n'est pas tant l'objet de la quête qui importe que le mouvement qu'elle génère, lequel oblige le sujet à se positionner par rapport à une histoire jamais donnée à voir autrement que comme idéalement syncrétique et pluriculturelle. La représentation de la ville en tant que lieu de partage et de coexistence pacifique a souvent prévalu dans l'imaginaire collectif. Le dernier roman d'Ángel Vázquez réussit à illustrer cette réalité historique et fictionnelle et révèle ses contradictions. Le défi du romancier s'annonce dès le prologue (ou l'avis au lecteur) qui revendique la portée testimoniale de l'œuvre, son intention d'être une « trace » de l'histoire, écho de sa propre mémoire qu'il va ainsi questionner avant que la ville ne « retourne à son passé arabe ${ }^{27} »$. Mais pour l'écrivain, c'est la langue,

26. La Vida perra, op. cit., p. 268. «Foutue mémoire! L'étagère, l'étagère... Ma pauvre tête! » (p. 346).

27. « Hoy la ciudad retorna a su pasado árabe y sería de incautos contradecir a la todopoderosa madre Historia » (La Vida perra, op. cit., p. 11). « De nos jours, la ville renoue avec son passé arabe, et seuls les imprudents pourraient prétendre barrer le chemin de notre mère toute-puissante, l'Histoire » (p. 21). On peut d'ailleurs s'interroger sur le sémantisme du mot qui assimile d'une certaine façon l'indépendance à un retour aux origines, ce qui est faux puisque le Maroc n'avait jamais existé véritablement dans ces frontières et avec ce régime politique. 
fruit des conflits de l'histoire mais aussi des échanges qui se produisent nécessairement dans un espace commun circonscrit géographiquement, qui peut le mieux rendre compte de la complexité d'une ville-monde. Si l'on considère la relation fondamentale existant entre la langue et l'espace, on voit comment la topologie s'inscrit dans l'épaisseur du texte pour le miner en profondeur. Les noms de lieux sont disséminés dans l'espace textuel comme dans l'espace urbain. Ils sont une façon de le délimiter mais sont aussi signes d'identité. Juanita nous invite à la suivre dans le dédale de sa ville. Elle décline les noms de rues, souvent les mêmes car elle ne s'éloigne jamais du cœur de la médina : en nommant son espace, elle le circonscrit et se l'approprie. Elle se rassure en le possédant symboliquement. La fonction référentielle de l'espace est évidente et tout Tangérois qui a vécu dans la zone internationale à l'époque reconnaît sans peine sa ville. Du Petit Socco au Grand $\mathrm{Socco}^{28}$, en passant par la rue Siaghins ${ }^{29}$, le boulevard Pasteur ou la place de France, la ville se dessine et prend forme. La façon dont l'espace est désigné se fait l'écho de la présence des différentes puissances présentes dans la ville et de leur volonté de la marquer de leur empreinte. La langue, sous toutes ses manifestations, est un instrument politique et un enjeu de pouvoir; mais si les ruelles de Tanger gardent l'empreinte de l'histoire de la ville, elles laissent aussi la place à l'imagination du lecteur, lequel se déplace à travers un espace poétique et fictionnel :

À aucun moment le lecteur n'a le sentiment que les plans de dénotation et de connotation opèrent un décrochage, qu'une lecture doive congédier ou exiger une autre lecture. Il en va de même dans le cas des nombreux noms de rues de Tanger [...]. Et pourtant quels merveilleux signifiants de connotation que tous ces noms de rues accumulés, embrouillés ou déroulés à plaisir [...]. Ils sont poésie pure, connotateurs du délire du narrateur, de son chaotique itinéraire spirituel, du soliloque inachevé ${ }^{30}$.

Le monologue rend compte d'un double trajet, à travers le temps et l'espace, et la différence entre les deux s'abolit dans les représentations qui en sont données. L'espace ne se laisse appréhender que dans l'instant présent, mais il s'inscrit dans une évolution qui lui confère sa pleine identité. Quant à la narratrice, elle continue à nommer, comme elle l'a toujours fait, les lieux de sa ville et par cette démarche, elle les consacre

28. «No, mujer, si no me cuesta ningún trabajo, te dejaré en el Zoco Grande » (La Vida perra, op. cit., p. 94). «Mais non, voyons. Ça ne me dérange pas du tout. Je te laisse au Grand Socco » (p. 123).

29. «En la vida he subido la cuesta de los Siaghins a esta velocidad» (La Vida perra, op. cit., p. 24). «Jamais de ma vie je n'ai remonté la rue des Siaghins à cette vitesse » (p. 34).

30. C'est ce que Bernard Loupias écrit du roman de Juan Goytisolo : «Importance et signification du lexique d'origine arabe dans Reivindicación del Conde don Julián de Juan Goytisolo », Bulletin hispanique, vol. 80, n 3-4, 1978, p. 238-239. 
définitivement comme des lieux de mémoire ${ }^{31}$. En figeant leur nom dans une époque révolue, elle dit ce qui fut, et non pas toujours ce qui est. En 1961, la plupart des noms de la ville ont été arabisés. Par exemple, la « calle de los cristianos » dont parle encore la narratrice est devenue la rue Cadi Ayad $^{32}$, cette transformation toponymique n'est cependant pas prise en compte par le discours. Il existe une logique diégétique à cet état de fait (une vieille femme, qui est en outre son seul interlocuteur, n'a aucune raison de changer ses habitudes) mais on voit ainsi comment l'écrivain déambule à travers sa propre mémoire lorsqu'il écrit ${ }^{33}$. Le temps de l'histoire et celui de la mémoire s'enchevêtrent parfois, il n'y a toutefois pas de relation mimétique entre les deux; la temporalité historique et objective se disloque dans le temps mémoriel qui est lui intime, subjectif et discontinu.

Les remarques décousues de Juanita Narboni laissent deviner que les différentes puissances qui se sont partagé la gestion de la ville ont organisé l'espace en fonction des besoins de leur communauté. Chacune possède son école, son hôpital ${ }^{34}$, ses lieux de culte ou ses cimetières ${ }^{35}$, voire ses commerces. La rue des Siaghins est sans doute un cas unique dans le monde où église catholique, synagogue et mosquée s'alignent pacifiquement comme pour mieux signifier une coexistence harmonieuse entre tous ${ }^{36}$. Après l'Indépendance, on assiste à un renversement des enjeux ainsi qu'à une relecture et à une déconstruction des significations. Ce qui se révèle dans les failles d'un discours nostalgique, mais parfois aussi lucide, c'est que l'espace partagé était aussi un espace fragmenté, un lieu d'exclusion et d'hégémonie. Pour Ángel Vázquez, c'est la spécificité d'une langue perméable à son environnement, une langue qui rend presque visibles

31. Nous reprenons l'expression désormais associée à Pierre Nora mais en lui donnant un sens quelque peu différent.

32. Dominique Pons, op.cit., p. 307.

33. Lorsque La Vida perra de Juanita Narboni est publié, en 1976, l'écrivain vit en Espagne depuis plus de dix ans et n'est jamais revenu dans sa ville.

34. «¿A qué hospital lo llevarán? Hospitales no faltan... ¿Al hospital Benchimol, al inglés, al italiano, al español...? » (La Vida perra, op. cit., p. 211). « À quel hôpital l'emmène-ton? Ce n'est pas ce qui manque les hôpitaux... L'hôpital Benchimol, les hôpitaux anglais, italiens, espagnol...? » (p. 272).

35. « ¿A dónde fueron a parar? La mayoría al cementerio. Bueno a los cementerios [...]. Los católicos en Bubana, los judíos repartidos entre el cementerio viejo y el nuevo, los protestantes en Saint Andrew, y esas negra de Hamruch [...] no creo que la hayan enterrado en Sidi Buarrakía » (La Vida perra, op. cit., p. 250). «Et où sont-ils? Au cimetière, pour la plupart. Enfin, dans un cimetière [...]. Les catholiques sont à Boubana, les Juifs dans l'ancien ou le nouveau cimetière, c'est selon, les protestants sont à Saint-Andrew et Amrouche, cette poison [...] je ne pense tout de même pas qu'elle soit à Sidi Bouarrakia » (p. 323).

36. Thierry de Beaucé dans La Chute de Tanger fait dire à son personnage que l'église de la Purísima était si bien intégrée dans son environnement qu' « on la prenait pour une mosquée » (p. 192). 
les strates de l'histoire de la communauté séfarade (migrations, contacts, ruptures et résistances ${ }^{37}$ ) qui sera sa voix et celle de « sa » ville. Le défi du romancier est d'assigner une portée presque universelle à une langue qui ne dépasse guère les limites d'une ville et qui a pratiquement disparu lorsque ceux qui la parlaient se sont dispersés. C'est sans doute cette langue en train de disparaître, qui n'était pas la sienne, mais qui évoquait le souvenir de sa mère ${ }^{38}$, qui pouvait le mieux dire la quête identitaire d'un homme et d'un écrivain. Cette langue exprime de plus la vérité d'une ville dans l'histoire. Langue exclusivement orale et vernaculaire, la hakitia devient dans La Vida perra de Juanita Narboni un langage poétique unique. Le lecteur se promène dans le texte en rencontrant des mots inconnus de lui au début mais qu'il finit par apprivoiser et qui lui deviennent familiers. Si pour un Tangérois qui connut les riches heures de la ville, cette langue hybride n'a rien de surprenant, elle garde cependant une partie de son mystère pour un lecteur hispanophone ${ }^{39}$. La langue devient le miroir d'une ville qui ne se livre jamais totalement. C'est sans doute pour cette raison qu'on n'a pas encore fini d'écrire, et donc de lire Tanger.

Nathalie Sagnes Alem

Université Paul-Valéry, Montpellier III

37. «Según los eruditos, en el yaquetía se entremezclan, a decir verdad con muchísimo salero, el castellano antiguo con el hebreo, salpicado de árabe y de portugués » (La Vida perra de Juanita Narboni, op. cit., prologue). «Pour les linguistes, c'est un mélange - hautement savoureux à vrai dire - d'ancien castillan et d'hébreu, métissé d'arabe et de portugais » (p. 20). En fait Vázquez en donne une définition quelque peu simplificatrice et incomplète.

38. Nous renvoyons à la dédicace : «En memoria de mi madre y de su tertulia de amigas, hebreas y cristianas, de cuyo lenguaje-recuerdo se apoderó Juanita Narboni, obligándome a escribir este libro. » («En mémoire de ma mère et de son cercle d'amies, juives et chrétiennes, dont Juanita Narboni s'est approprié le langage-souvenir, m'obligeant à écrire ce livre », p. 7).

39. Et on peut même ajouter pour un lecteur francophone, grâce à l'excellente traduction de Selim Cherief. 